\title{
Core Outcome Sets (COS) related to pregnancy and childbirth: a systematic review
}

Marie Österberg ${ }^{1 *}$, Christel Hellberg ${ }^{1}$, Ann Kristine Jonsson ${ }^{1}$, Sara Fundell ${ }^{1}$, Frida Trönnberg ${ }^{2}$, Alkistis Skalkidou ${ }^{3}$ and Maria Jonsson ${ }^{3}$

\begin{abstract}
Background: Systematic reviews often conclude low confidence in the results due to heterogeneity in the reported outcomes. A Core Outcome Set (COS) is an agreed standardised collection of outcomes for a specific area of health. The outcomes included in a COS are to be measured and summarized in clinical trials as well as systematic reviews to counteract this heterogeneity.
\end{abstract}

Aim: The aim is to identify, compile and assess final and ongoing studies that are prioritizing outcomes in the area of pregnancy and childbirth.

Methods: All studies which prioritized outcomes related to pregnancy and childbirth using consensus method, including Delphi surveys or consensus meetings were included. Searches were conducted in Ovid MEDLINE, EMBASE, PsycINFO, Academic Search Elite, CINAHL, SocINDEX and COMET databases up to June 2021.

For all studies fulfilling the inclusion criteria, information regarding outcomes as well as population, method, and setting was extracted. In addition, reporting in the finalized studies was assessed using a modified version of the Core Outcome Set-STAndards for Reporting.

Results: In total, 27 finalized studies and 42 ongoing studies were assessed as relevant and were included. In the finalized studies, the number of outcomes included in the COS ranged from 6 to 51 with a median of 13 outcomes. The majority of the identified COS, both finalized as well as ongoing, were relating to physical complications during pregnancy.

Conclusion: There is a growing number of Core Outcome Set studies related to pregnancy and childbirth. Although several of the finalized studies follow the proposed reporting, there are still some items that are not always clearly reported. Additionally, several of the identified COS contained a large number $(n>20)$ outcomes, something that possibly could hinder implementation. Therefore, there is a need to consider the number of outcomes which may be included in a COS to render it optimal for future research.

Keywords: Core outcome set, Consensus methods, Maternal health, Obstetric care, Pregnancy, Prenatal

\footnotetext{
*Correspondence: Marie.Osterberg@sbu.se

${ }^{1}$ Swedish Agency for Health Technology Assessment and Assessment

of Social Services (SBU), Stockholm, Sweden

Full list of author information is available at the end of the article
} original author(s) and the source, provide a link to the Creative Commons licence, and indicate if changes were made. The images or other third party material in this article are included in the article's Creative Commons licence, unless indicated otherwise in a credit line to the material. If material is not included in the article's Creative Commons licence and your intended use is not permitted by statutory regulation or exceeds the permitted use, you will need to obtain permission directly from the copyright holder. To view a copy of this licence, visit http://creativecommons.org/licenses/by/4.0/. The Creative Commons Public Domain Dedication waiver (http://creativeco mmons.org/publicdomain/zero/1.0/) applies to the data made available in this article, unless otherwise stated in a credit line to the data. 


\section{Background}

Well- designed and conducted clinical trials, mainly randomised controlled trials (RCTs), are used to establish the effectiveness of different interventions through comparison of outcomes. However, when research results are later synthesised in systematic reviews, it becomes clear that studies often overlook outcomes of importance to patients, that different outcomes are assessed and that different methods or timepoints for assessment are used. This has a negative impact on the certainty of the findings in systematic reviews, thus contributing to research waste. As a result, the scientific evidence to support many treatment procedures is attenuated $[1,2]$.

To overcome these problems, the core selection of outcomes and measurement properties in studies need to be standardised. Described and promoted by the Core Outcome Measures in Effectiveness Trials (COMET) initiative group in 2010, Core Outcome Sets (COS) have increasingly been developed for various conditions over time. According to COMET "A COS is a minimum set of outcomes to be selected, measured, and reported in trials of a specific condition" [3]. These are typically developed by identifying and describing the outcomes used in current research (primary studies as well as systematic reviews) and then allowing stakeholders to prioritize among these outcomes by using a consensus process. When a COS has been agreed on, the purpose is that researchers use it in all studies within that condition, adding further outcomes if they wish.

The aim of developing and implementing COS is that the results of various studies will be more readily comparable and collated, reinforcing the basis of decisions, to benefit patients and healthcare personnel.

In the research fields of women's health and neonatal health, an international network, called CoRe Outcomes in Women's and Newborn health (CROWN), has been established [4]. It is led by journal editors, and aims to address the widespread, unwarranted variation in reporting of outcomes, which makes comparison between and combination of results across studies difficult, if not impossible. This initiative might explain the rather large production of COS in this area. This was also illustrated in a previous systematic review with focus on COS related to the health of women and new-born published in 2017 [5]. This review identified four finalized COS and an additional 49 ongoing COS, thus motivating an updated systematic review to investigate any new activity on the topic.

The aim of this article is to systematically identify and describe ongoing and finalized COS projects (including all projects where outcomes where prioritized), within the field of pregnancy and childbirth.

\section{Methods}

The study consisted of a systematic literature review undertaken to analyse and summarize ongoing and finalized COS projects (including all projects where outcomes where prioritized), within the area of pregnancy and childbirth. The literature search was conducted in June 2019, and an updated search was conducted in June 2021.

\section{Protocol and registration}

This manuscript is an updated version of a governmental report published by SBU 2020 [6].

A project plan was established a priori and registered at SBU, the PROSPERO database (CRD420201490792020)

[7] as well as the COMET database [8]. This systematic review was conducted and reported in accordance with the PRISMA statement [9].

\section{Eligibility criteria}

The criteria for eligibility were outlined according to the PICOS model (Population, Intervention, Comparator, Outcome and Study design) and included the following characteristics:

\section{Population}

Pregnant women, women during labour and birth, women who suffer from an injury or other complications related to childbirth, women or men suffering from a mental health disorder during pregnancy or during or after childbirth.

\section{Intervention}

No restriction.

\section{Control}

Not applicable.

\section{Outcome}

A list of outcomes included in the COS.

\section{Study design}

Ongoing or finalized original studies where outcomes were prioritized using some form of consensus. No restriction applied to publication status.

\section{Language}

English and Scandinavian languages.

\section{Exclusion criteria}

- Systematic reviews of outcomes

- Qualitative studies identifying important outcomes, without any form of prioritization 
- COS studies focusing only on the child (no outcomes related to the women)

- COS studies relating to interventions/conditions prior to pregnancy, such as in vitro fertilization, contraceptives use etc.

\section{Information sources and search strategy}

Studies were identified by searching electronic databases and by scanning the reference lists of studies meeting the eligibility criteria. The electronic databases MEDLINE, Embase, PsycINFO, Academic Search Elite, CINAHL with Full Text and SocINDEX with Full Text and the Core Outcome Measures in Effectiveness Trials (COMET) Initiative database were searched up to June 2021. Electronic searches were conducted using a combination of medical subject headings $(\mathrm{MeSH})$ and relevant text word terms related to the population, in combination with different terms related to Core Outcome Set. (For detailed information about the search strategies, Additional file 1) In addition, the CROWN website was hand searched [4].

\section{Identification of studies}

Two reviewers ( $\mathrm{MÖ}$ and $\mathrm{CH}$ ) independently screened the titles and abstracts for eligibility. The abstracts were screened and rated using the scanning tool Rayyan, available online [10]. Full text articles were retrieved and reviewed to determine eligibility, independently and in duplicate by two authors ( $\mathrm{CH}$ and MÖ). Disagreements were resolved by discussion. The reference lists of studies meeting the eligibility criteria were screened for additional relevant studies.

\section{Description of methodology in included studies}

In order to check the description of the methodology in the included studies, a checklist was compiled using the items from the COS-STAR reporting guide (Additional file 2). The COS-STAR is developed as a reporting checklist and is not developed or validated as a quality assessment tool [11]. However, no such tool exists, and the project management team decided to use this existing reporting guide to investigate the COS. The involvement of relevant stakeholders is an important feature of COS development; therefore, one further question was added to the checklist: "Are researchers as well as healthcare providers and patients included in the development process?" (Additional file 2). Two of the authors $(\mathrm{CH}$ and $\mathrm{MO}$ ) independently reviewed the included articles according to the checklist. Disagreements were resolved by discussion.

\section{Data items}

The following information was extracted from the included trials: Population, intervention, setting for intended use, consensus method, number and characteristics of participants, number of outcomes at the start of the project and number of outcomes in the final COS, consensus criteria and the degree of compliance with COS-STAR.

Data were extracted from each included study and tabled by one reviewer. A second reviewer audited the data extraction. Any disagreements were resolved by discussion.

Since the results were not suitable for synthesis, the included studies are described narratively.

\section{Patient involvement}

A patient representative with lived experience of birth trauma, birth injury and postpartum depression (FT) was included in the project management group to ensure patient input into all aspects of the work.

\section{Results \\ Eligible studies}

The literature search yielded a total of 3334 citations: after review of the abstracts, 154 were assessed in full. Eighty-five studies which did not meet the inclusion criteria were excluded, leaving 69 relevant studies. Of these, 27 [12-38] were finalized studies with prioritized outcomes and 42 were COS protocols, where the final COS was not yet published (Fig. 1) [39-80].

Information about the included finalized COS studies is presented in Table 1 and the outcomes included in the final COS are presented in Table S3. Excluded studies and the reason for exclusion can be found in Table S1. Forty of the 42 ongoing COS studies were identified through the COMET database and a published full protocol was identified for 14. The ongoing studies are described in Table S2.

\section{Published core outcome sets}

In total, 19 of the finalized studies had COS development as the main purpose $[12,15,16,18,19,22,26,30,31,34$, $37,38]$ (Table 1). Of the eight remaining included studies, the main aim of the studies varied somewhat, but they all included prioritization of outcomes [14, 17, 23, 24, 32, $33,35,36]$. Two articles had as primary aim to prioritize future research questions, including prioritizing the outcomes to be measured [14, 36]. Two articles examined which outcomes to include in a composite outcome and other studies considered outcomes to be included and assessed in clinical follow-up of patients or reporting to registers $[17,23,24,32,33,35]$. Six of the 27 studies were 


\section{Records identified through database searching} 3168

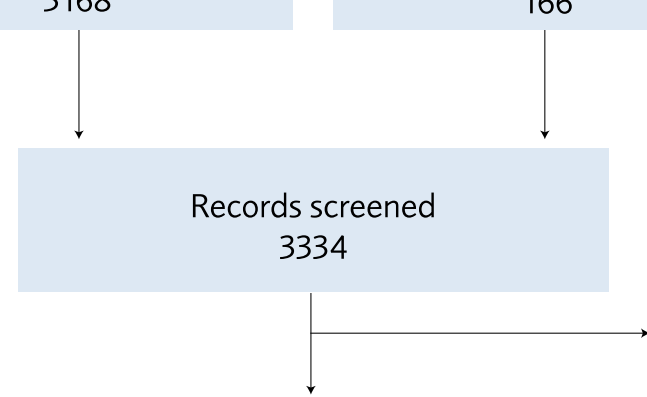

Full-text articles assessed for eligibility 154

Finished COS 27

\section{Eligible full-text articles}

69
Additional records identified through COMET database

166

Excluded records 3180

Excluded articles

85

Fig. 1 Study flow diagram

not registered in the COMET databases (Table 1). Of those registered only two did not yet provide a link to the published paper [27, 37].

Of the finalized studies, all were published after 2007 and $67 \%$ were published during 2018-2021 (Fig. 2A). The large number of ongoing COS projects identified indicates a high degree of activity in the field.

Categorisation of studies (Fig. 2B), disclosed that most COS, both finalized and ongoing, focus on pregnancy and pregnancy-related complications and conditions. There are few COS studies focusing on labour, birth and physical conditions associated with giving birth. Also, a limited number of studies, one finalized and two ongoing, were identified relating to mental health during pregnancy or after childbirth [27, 39, 49]. Since the focus of our review is Core Outcome Sets relating to pregnancy and childbirth and not neonatal and fetal aspects only a few finalized COS have been included which presents both outcomes related to the women as well as the featus/ newborn. Therefore, the number of finalized COS relating to fetal/neonatal should be interpreted with caution.
And we are aware of at least two additional finalized COS which were excluded from this review since the focus is on the new-born [81, 82].

\section{Use of method and representation}

Most of the finalized studies described a 2 or 3 round Delphi survey, followed by a face-to-face consensus meeting to finalize the COS (Table 1). However, some finalized studies included only Delphi surveys and one study by Fiala et al. only undertook a consensus-meeting (Table 1) [23].

The consensus criteria most commonly used for an outcome to be included in the COS was the "70/15 rule" (more than $70 \%$ rates the outcome as critically important and less than $15 \%$ rates it as not important) (Table 1). The number of outcomes included in the COS ranged between 6 and 51 with a median number of 13 (Fig. 2C, Table 1). Only a few studies had less than 10 outcomes in the final COS. Only one study mentioned that a possible maximum limit to the number of outcomes to be included in the COS had 


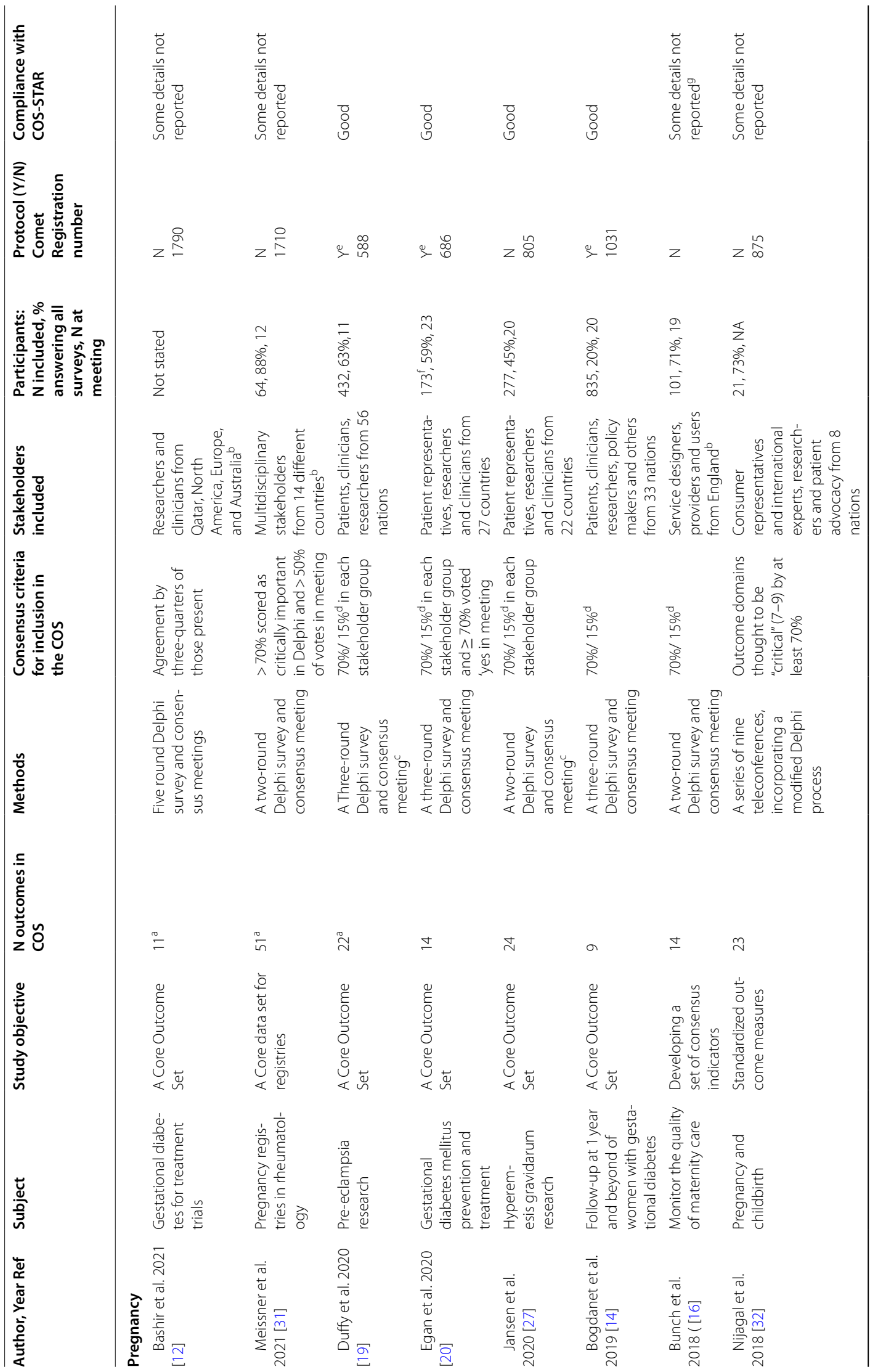




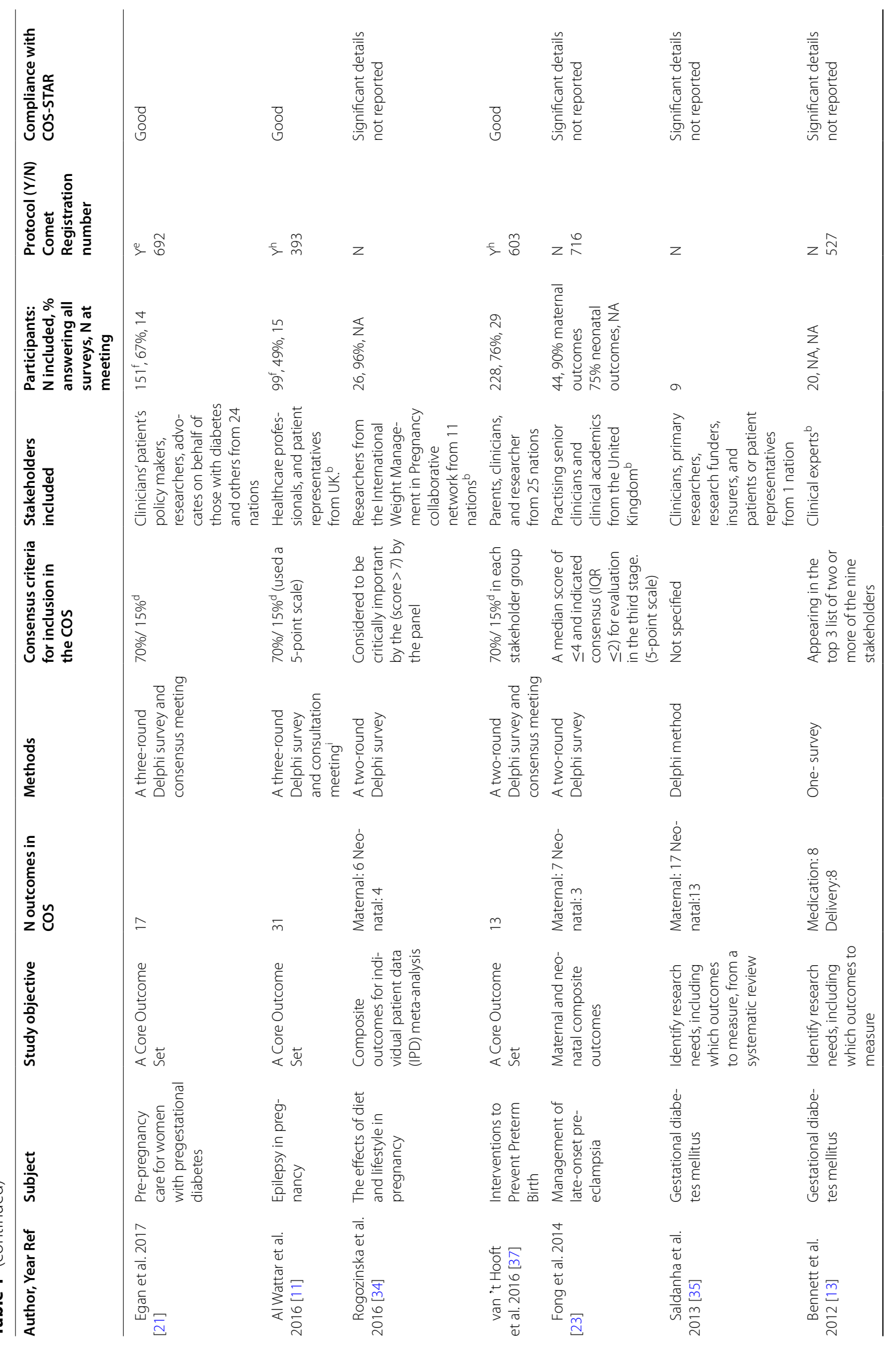




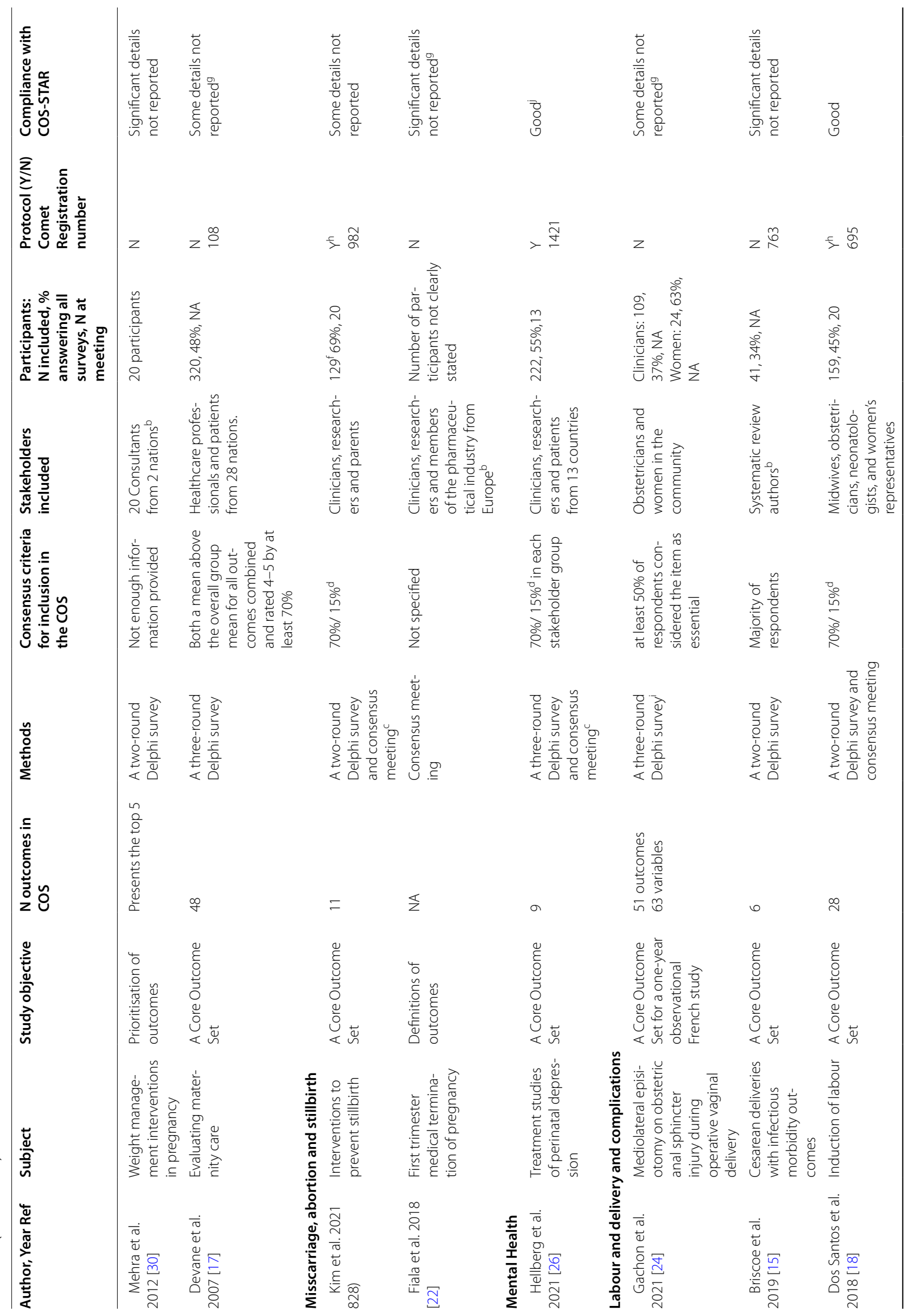




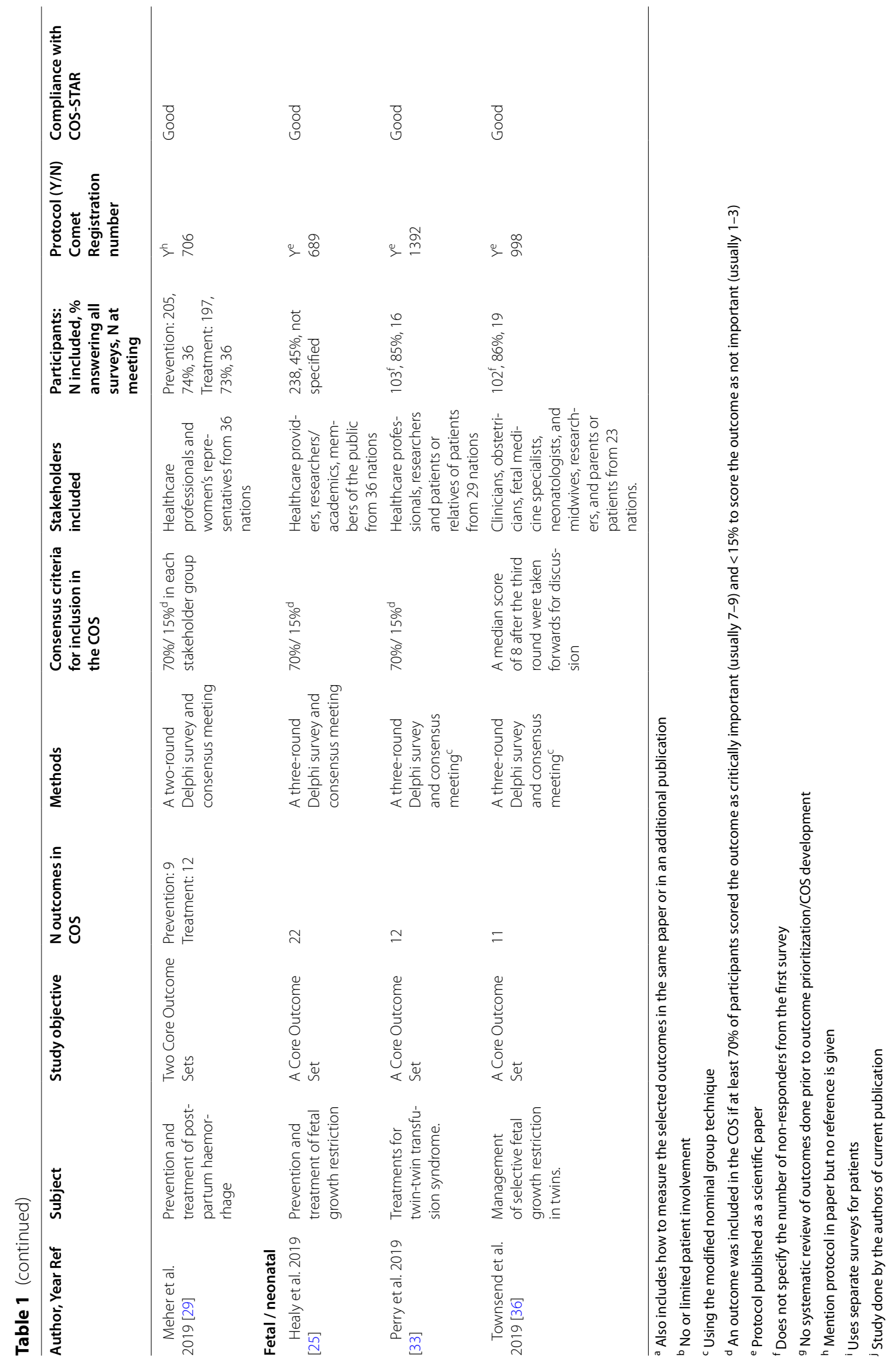




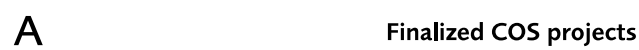

6

5

4

3

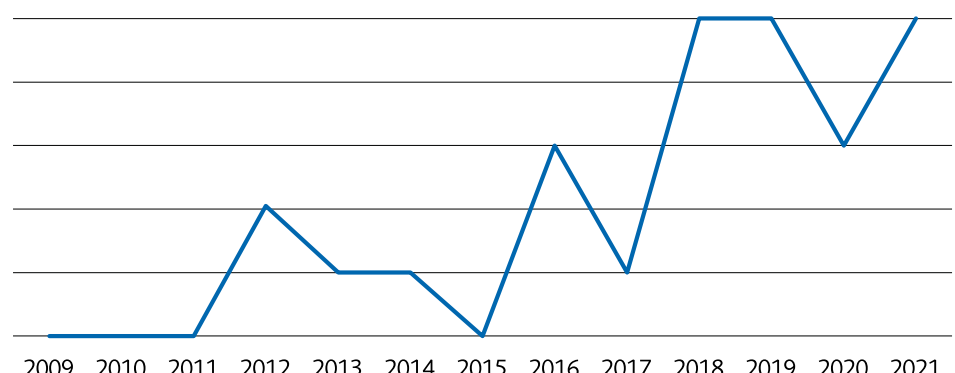

$20092010201120122013201420152016 \quad 20172018 \quad 201920202021$

B Finialized and ongoing projects

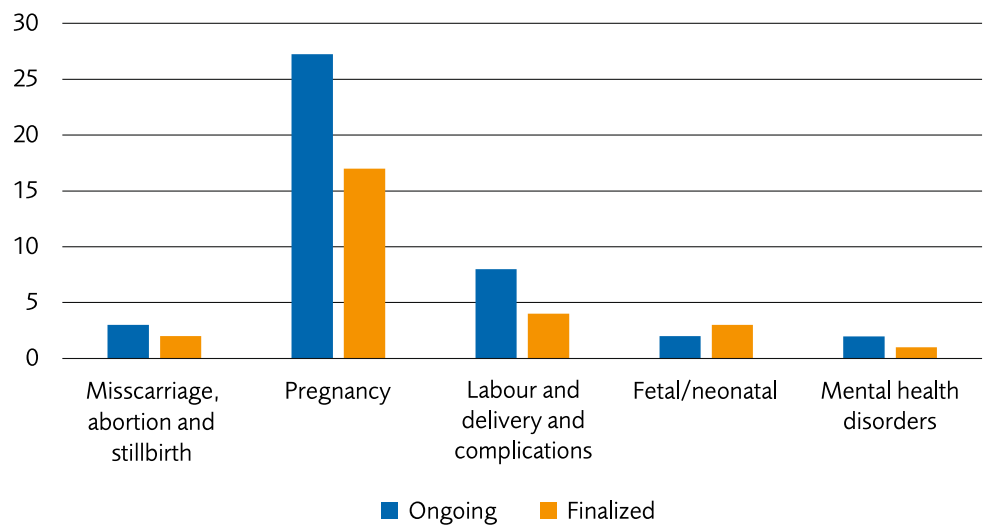

C Number of outcomes included in finalized $\cos$

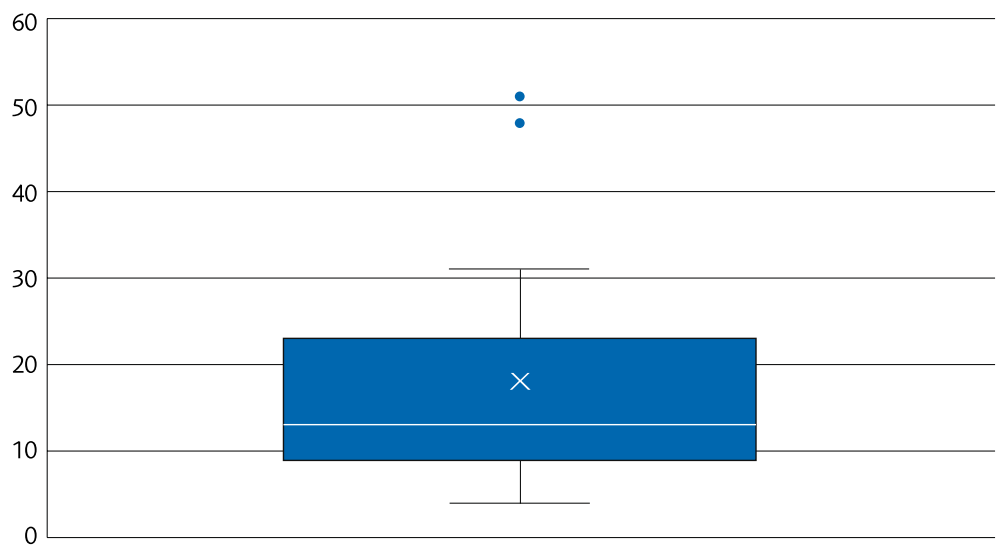

Fig. 2 Description of included final and ongoing COS studies. A Number of final COS studies by year of publication. B Number of final and ongoing COS studies categorized by sub-topics and C Boxplot depiction of number of outcomes in the final COS (median 13 and mean 18). Data from studies with the aim of prioritizing which outcomes to include in a composite outcome or only presenting the top outcomes in the COS are not included in the boxplot

been determined or discussed in advance, in order to enable implementation and feasibility in research [27]. Six studies described using a "modified nominal group technique" during the consensus meeting in order to reduce the number of outcomes (Table 1) [20, 27-29, $34,37]$. 
Researchers were included in all identified studies and healthcare personnel in the majority of them. Patients were sometimes not included at all in the process or only partly included [12-14, 16, 17, 23, 24, 31, 32, 35]. Some examples of limited patient inclusion: Al Wattar et al. [12] who used a separate survey consisting of only one round for patients; Bunch et al. [17] where patients were included in the Delphi survey, but not in the consensus meeting and Bennet et al. [14] where two persons served as proxies for patients. Most of the finalized studies involved international participation (Table 1).

Thirteen of the studies were assessed as complying well with the COS-STAR criteria in most categories [12, 15 , 19-22, 26-28, 30, 34, 37, 38], seven showed some deviations $[13,17,18,25,29,32,33]$ and seven of the studies were assessed as having major shortcomings in reporting $[14,16,23,24,31,35,36]$; however, five of those were published prior to the publication of COS-STAR (Table 1 and Table S4). Most of the finalized studies lacked information about whether outcomes had been excluded at some stage or if outcomes had been merged. Only one study mentioned whether they deviated from the study protocol in any way [27].

\section{Discussion}

\section{Main findings}

Although there are examples of well-established COS such as Outcome Measures in Rheumatology (OMER$\mathrm{ACT})$ for rheumatoid arthritis, they are still relatively rare in most medical fields [83]. This review of pregnancy and childbirth revealed that most of the COS are developed for physical conditions that occur during pregnancy. A minority of the ongoing or existing COS focus on mental health. There are also a few COS on intrapartum care, for such conditions as slow progress in labour, trial of labour after previous caesarean section and postpartum endometritis. One of the topics for which most COS have been compiled is the field of physical conditions and complications during pregnancy.

It is important to consider how many outcomes a COS can include and still be applicable and useful for research. This systematic review discloses that the COS identified range between 6 and 51 outcomes with a median of 13 outcomes. Only a few of the included finailzed COS had less than ten outcomes. None of the identified studies discussed the relationship between the number of outcomes in the COS and the median number of outcomes in the studies for which the COS is intended for. Nor did any of the protocols suggest a possible maximum limit to the number of outcomes that might be included in the intended COS. In order to increase the implementation of the developed COS, it is important to consider how the number of outcomes included will affect the usefulness of the COS. A limitation of the number of outcomes might increase the likelihood that the COS are indeed applied in future research. There are consensus processes that include several stakeholders, where a preset goal is communicated from start to the participants. The prioritization of research questions by James Lind Alliance is one example of such a process, where a topten list of research questions are to be agreed upon [84]. Surely, such a limit might impact the COS development process and it might be even more important to balance the influence between different stakeholders along the process, especially in consensus meetings. Nevertheless, a limit might also be a positive contributing factor in the process, putting pressure on the participants to limit their choices of the most important outcomes.

Another aspect that might need further discussions and guidance is how extensive the scope of a COS can be. As illustrated in this systematic review, some COS are more generic, covering broad areas, as for example the whole maternal care period, while others are more precise and niched, as for example twin to twin transfusion syndrome. This might result in numerous overlapping COS, and potentially introduces challenges when researchers are faced with more than one COS to comply with.

It is also important to note that the development of a COS which focuses on what to measure may need to be followed by decisions about how and when to measure these outcomes. Even if the outcomes themselves are consistent across the studies, lack of consistency in how or when outcomes have been measured can undermine efforts by systematic reviewers to compare, contrast and combine the results of multiple studies. Unfortunately, only a few of the identified COS mentioned how and when to measure the outcomes in the developed COS.

\section{Strengths and limitations}

Some limitations to the systematic review should be noted. In the systematic review, we checked compliance to COS-STAR in the included studies (Additional file 2) [11]. Another possibility would have been to check how well the different projects adhered to the COS-STAD guidelines [85]. However, none of these guidelines was developed to check methodological quality. For instance, both recommendations discuss that one should describe/ report a scoring process and consensus definition a priori, but not if the process/definition was suitable. In addition, the COS-STAD does not include items concerning the availability of a protocol, if any adjustments were made to it, or if conflicts of interest and ethical approval existed [85]. This guided our decision to check for how well the published studies reported their findings in accordance with COS-STAR. In addition, we also checked if all relevant stakeholder groups were included 
in the development. However, it would have been optimal to be able to assess the methodological quality of the included studies using a tool developed for this purpose. We believe that the development of such a tool is desirable and that some of the questions used in this article (Additional file 2) could be helpful. Further, in this systematic review, we decided to have an inclusive approach and might have included studies that were not principally intended for research use, but for other purposes, such as clinical follow-up.

A strength of this study is that it is methodologically sound and robust, and all results have continuously been reviewed by experts from the Swedish Agency for Health Technology Assessment and Assessment of Social Services (SBU), as well as by external reviewers. Another strength is the attempt to assess the reporting of the included COS using an assessment tool based on the COS-STAR reporting guide (Additional file 2).

\section{Interpretation}

In 2017, Duffy et al. published a systematic review of published and ongoing COS related to the health of women and newborns [5]. The scope of their paper is somewhat broader, including conditions other than those related to pregnancy and childbirth. In all, they identified four finalized COS, of which three were related to pregnancy and childbirth. In the last years, a substantial number of COS have been finalized and 42 ongoing studies have been identified.

\section{Conclusion}

This systematic review discloses an increasing number of COS for pregnancy and childbirth. This is gratifying and is hopefully leading to studies which focus on important outcomes and research that are more readily synthesised in systematic reviews, thus increasing evidence in support of interventions. The review reveals that a large number of the ongoing and finalized COS studies address physical conditions and complications during pregnancy. There was a lack of COS for birth-related studies. Only a few COS were identified for perinatal mental health.

\footnotetext{
Abbreviations

COMET: Core Outcome Measures in Effectiveness Trials Initiative; COS: Core Outcome Set/ Sets; COS-STAD: The Core Outcome Set-STAndards for Development; COS-STAR: Core Outcome Set-STAndards for Reporting; CROWN: Core Outcomes in Women's and Newborn Health; RCT : Randomised Controlled Trial; SBU : Swedish agency for health technology assessment and assessment of social services; HTA: Health Technology Assessment.
}

\section{Supplementary Information}

The online version contains supplementary material available at https://doi. org/10.1186/s12884-021-04164-y.

Additional file 1. Search strategies.

Additional file 2. Checklist for included studies.

Additional file 3: Table S3. Included studies.

Additional file 4: Table S1. Excluded studies.

Additional file 5: Table S2. Ongoing COS studies.

Additional file 6: Table S4. Compliance with COS-STAR Items.

\section{Acknowledgements}

Not applicable.

Tweetable abstract

A systematic review of finalized and ongoing Core Outcomes Sets (COS) relating to pregnancy and childbirth by @SBU_en.

\section{Authors' contributions}

Study concept and design: CH, MÖ, AS, MJ, FT. Literature search AJ. Selection of studies and extraction of the relevant information $\mathrm{CH}$ and $\mathrm{MÖ}$. Analysis and interpretation of data: $\mathrm{CH}, \mathrm{MÖ}, \mathrm{AS}, \mathrm{MJ}$, FT. Drafting of the manuscript: $\mathrm{CH}$ and $M O ̈$. Critical revision of the manuscript for important intellectual content: AS, MJ, FT, CH, MÖ, SF, AJ. The author(s) read and approved the final manuscript.

\section{Authors' information}

MÖ, CH, SF, AJ are employed by the national Swedish HTA agency SBU, this article is adapted from a report undertaken by the SBU, who provided funding for the study.

\section{Funding}

The project was conducted within the Swedish Agency for Health Technology Assessment and Assessment of Social Services assignment, external funding was not sought or used.

Availability of data and materials

The datasets used and/or analysed during the current study are available from the corresponding author on reasonable request.

\section{Declarations}

Ethics approval and consent to participate Not applicable.

Consent for publication

Not applicable.

\section{Competing interests}

The authors of this review are also the authors of one of the included finalized COS study. The authors report no additional conflict of interest, all authors filed a conflicts of interest form used by Swedish governmental agencies before engagement. These are available upon request.

\section{Author details \\ ${ }^{1}$ Swedish Agency for Health Technology Assessment and Assessment of Social Services (SBU), Stockholm, Sweden. ${ }^{2}$ Patient Representative, Linköping, Sweden. ${ }^{3}$ Department of Women's and Children's Health, Uppsala University, Uppsala, Sweden.}

Received: 25 March 2021 Accepted: 24 September 2021

Published online: 09 October 2021 


\section{References}

1. Clarke M, Williamson PR. Core outcome sets and systematic reviews. Syst Rev. 2016;5(1):11.

2. Williamson PR, Altman DG, Bagley H, Barnes KL, Blazeby JM, Brookes ST, et al. The COMET handbook: version 1.0. Trials. 2017;18(3):280.

3. COMET Initiative. Core Outcome Measures for Effectiveness Trials (COMET) initiative. Available from: https://www.comet-initiative.org/. Cited 2020 Nov 24.

4. The Core Outcomes in Women's and Newborn health (CROWN). Available from: http://www.crown-initiative.org/. Cited 2020 Jan 30.

5. Duffy J, Rolph R, Gale C, Hirsch M, Khan KS, Ziebland S, et al. Core outcome sets in women's and newborn health: a systematic review. BJOG. 2017;124(10):1481-9.

6. SBU. Core outcome sets inom förlossningsvård. Sammanställning och analys av studier. Stockholm: Statens beredning för medicinsk och social utvärdering (SBU); 2020. SBU-rapport nr 309. ISBN 978-91-88437-51-8

7. Hellberg C, Österberg M, Jonnson M, Skalkidou A, Ann Kristine Jonsson. A systematic review over core outcomes sets (COS) in obstetric care. PROSPERO: international prospective register of systematic reviews. 2020 CRD420201490792020. Available from: https://www.crd.york.ac.uk/PROSP ERO/display_record.php?RecordID $=149079$.

8. COMET Initiative. COMET database. A systematic review over core outcome sets in obstetric care. Available from: http://www.comet-initiative. org/Studies/Details/1370. Cited 2020 Jan 30.

9. Page MJ, McKenzie JE, Bossuyt PM, Boutron I, Hoffmann TC, Mulrow CD, et al. The PRISMA 2020 statement: an updated guideline for reporting systematic reviews. BMJ. 2021;372:n71.

10. Ouzzani M, Hammady H, Fedorowicz Z, Elmagarmid A. Rayyan-a web and mobile app for systematic reviews. Syst Rev. 2016:5(1):210.

11. Kirkham JJ, Gorst S, Altman DG, Blazeby JM, Clarke M, Devane D, et al. Core outcome set-STAndards for reporting: the COS-STAR statement. PLoS Med. 2016;13(10):e1002148.

12. Al Wattar BH, Tamilselvan $K$, Khan R, Kelso A, Sinha A, Pirie AM, et al. Development of a core outcome set for epilepsy in pregnancy (E-CORE): a national multi-stakeholder modified Delphi consensus study. BJOG. 2017;124(4):661-7.

13. Bashir M, Syed A, Furuya-Kanamori L, Musa OAH, Mohamed AM, Skarulis $\mathrm{M}$, et al. Core outcomes in gestational diabetes for treatment trials: the Gestational Metabolic Group treatment set. Obes Sci Pract. 2021;7(3):251-9.

14. Bennett WL, Robinson KA, Saldanha IJ, Wilson LM, Nicholson WK. High priority research needs for gestational diabetes mellitus. J Women's Health (2002). 2012;21(9):925-32.

15. Bogdanet D, Reddin C, Macken E, Griffin TP, Fhelelboom N, Biesty L, et al. Follow-up at 1 year and beyond of women with gestational diabetes treated with insulin and/or oral glucose-lowering agents: a core outcome set using a Delphi survey. Diabetologia. 2019;62(11):2007-16.

16. Briscoe KE, Haas DM. Developing a core outcome set for cesarean delivery maternal infectious morbidity outcomes. Am J Perinatol. Am J Perinatol. 2020;37(4):436-52

17. Bunch KJ, Allin B, Jolly M, Hardie T, Knight M. Developing a set of consensus indicators to support maternity service quality improvement: using core outcome set methodology including a Delphi process. BJOG. 2018;125(12):1612-8.

18. Devane D, Begley CM, Clarke M, Horey D, Oboyle C. Evaluating maternity care: a core set of outcome measures. Birth (Berkeley, Calif) 2007;34(2):164-72.

19. Dos Santos F, Drymiotou S, Antequera Martin A, Mol BW, Gale C, Devane $D$, et al. Development of a core outcome set for trials on induction of labour: an international multistakeholder Delphi study. BJOG. 2018;125(13):1673-80.

20. Duffy JMN, Cairns AE, Richards-Doran D, van 't Hooft J, Gale C, Brown $\mathrm{M}$, et al. A core outcome set for pre-eclampsia research: an international consensus development study. BJOG Int J Obstet Gynaecol. 2020;127(12):1516-26.

21. Egan AM, Bogdanet D, Griffin TP, Kgosidialwa O, Cervar-Zivkovic $M$, Dempsey $E$, et al. A core outcome set for studies of gestational diabetes mellitus prevention and treatment. Diabetologia. 2020;63(6):1120-7.

22. Egan AM, Galjaard S, Maresh MJA, Loeken MR, Napoli A, Anastasiou E, et al. A core outcome set for studies evaluating the effectiveness of prepregnancy care for women with pregestational diabetes. Diabetologia. 2017;60(7):1190-6.

23. Fiala C, Cameron S, Bombas T, Parachini M, Agostini A, Lertxundi R, et al. Outcome of first trimester medical termination of pregnancy: definitions and management. Eur J Contracept Reprod Health Care. 2018;23(6):451-7

24. Fong F, Rogozinska E, Allotey J, Kempley S, Shah DK, Thangaratinam S Development of maternal and neonatal composite outcomes for trials evaluating management of late-onset pre-eclampsia. Hypertens Pregnancy. 2014;33(2):115-31.

25. Gachon B, Schmitz T, Artzner F, Parant O, De Tayrac R, Ducarme G, et al. A core outcome set development for a French national prospective study about the effect of mediolateral episiotomy on obstetric anal sphincter injury during operative vaginal delivery (INSTRUMODA). BMC Pregnancy Childbirth. 2021;21(1):251

26. Healy P, Gordijn SJ, Ganzevoort W, Beune IM, Baschat A, Khalil A, et al. A Core Outcome Set for the prevention and treatment of fetal GROwth restriction: deVeloping Endpoints: the COSGROVE study. Am J Obstet Gynecol. 2019;221(4):339.e1-.e10.

27. Hellberg C, Osterberg M, Jonsson AK, Fundell S, Tronnberg F, Jonsson $M$, et al. Important research outcomes for treatment studies of perinatal depression: systematic overview and development of a core outcome set. BJOG. 2021

28. Jansen L, Koot MH, Van't Hooft J, Dean CR, Duffy J, Ganzevoort W, et al. A core outcome set for hyperemesis gravidarum research: an international consensus study. BJOG. 2020;127(8):983-92.

29. Kim BV, Aromataris EC, Middleton P, Townsend R, Thangaratinam S, Duffy $J M N$, et al. Development of a core outcome set for interventions to prevent stillbirth. Aust N Z J Obstet Gynaecol. 2021.

30. Meher S, Cuthbert A, Kirkham JJ, Williamson P, Abalos E, Aflaifel N, et al. Core outcome sets for prevention and treatment of postpartum haemorrhage: an international Delphi consensus study. BJOG. 2019;126(1):83-93.

31. Mehra $\mathrm{H}$, Thangaratinam S. Prioritisation of outcomes in the evaluation of weight management interventions in pregnancy: a DELPHI survey. Arch Dis Child Fetal Neonatal Ed. 2012;97:A38.

32. Meissner Y, Fischer-Betz R, Andreoli L, Costedoat-Chalumeau N, De Cock $D$, Dolhain $R$, et al. EULAR recommendations for a core data set for pregnancy registries in rheumatology. Ann Rheum Dis. 2021;80(1):49-56.

33. Nijagal MA, Wissig S, Stowell C, Olson E, Amer-Wahlin I, Bonsel G, et al. Standardized outcome measures for pregnancy and childbirth, an ICHOM proposal. BMC Health Serv Res. 2018;18(1):953.

34. Perry H, Duffy JMN, Reed K, Baschat A, Deprest J, Hecher K, et al. Core outcome set for research studies evaluating treatments for twin-twin transfusion syndrome. Ultrasound Obstet Gynecol. 2019;54(2):255-61.

35. Rogozinska E, D'Amico Ml, Khan KS, Cecatti JG, Teede H, Yeo S, et al. Development of composite outcomes for individual patient data (IPD) meta-analysis on the effects of diet and lifestyle in pregnancy: a Delphi survey. BJOG Int J Obstet Gynaecol. 2016;123(2):190-8.

36. Saldanha IJ, Wilson LM, Bennett WL, Nicholson WK, Robinson KA. Development and pilot test of a process to identify research needs from a systematic review. J Clin Epidemiol. 2013;66(5):538-45.

37. Townsend R, Duffy JMN, Sileo F, Perry H, Ganzevoort W, Reed K, et al. A core outcome set for studies investigating the management of selective fetal growth restriction in twins. Ultrasound Obstet Gynecol. 2019.

38. van't Hooft J, JMN D, Daly M, Williamson PR, Meher S, Thom E, et al. A core outcome set for evaluation of interventions to prevent preterm birth. Obstet Gynecol. 2016;127(1):49-58.

39. COMET Initiative. COMET Database. Core Outcome Sets for Mental health following Early Pregnancy loss (COSMEP). Available from: https://www. comet-initiative.org/Studies/Details/1763. Cited 2021 Jun 10.

40. COMET Initiative. COMET Database. Development of a core outcome set for effectiveness studies of breech birth at term (Breech-COS): an international multi-stakeholder Delphi study. Available from: https://www. comet-initiative.org/Studies/Details/1749. Cited 2021 Jun 10.

41. COMET Initiative. COMET Database. CRADLE Delphi study: expert Consensus Regarding core outcomes for enhAnced recovery after cesarean DeLivery studiE. Available from: https://www.comet-initiative.org/Studi es/Details/1728. Cited 2021 Jun 10.

42. COMET Initiative. COMET Database. Core outcome set for studies of pregnancy affected by multimorbidity. Available from: https://www. comet-initiative.org/Studies/Details/1724. Cited 2021 Jun 10. 
43. COMET Initiative. COMET Database. Development and validation of a Midwife-led core outcome set (M-COS) for healthy women and babies. Available from: https://www.comet-initiative.org/Studies/Details/1723. Cited 2021 Jun 10.

44. COMET Initiative. COMET Database. Essential outcomes to be reported in all obstetric studies. Available from: https://www.comet-initiative.org/ Studies/Details/1719. Cited 2021 Jun 10.

45. COMET Initiative. COMET Database. Development of a core outcome set for diagnostic and therapeutic studies of urinary tract infection in pregnancy. Available from: https://www.comet-initiative.org/Studies/Details/ 1683. Cited 2021 Jun 10.

46. COMET Initiative. COMET Database. A core outcome set for COVID-19 and emerging pathogens in pregnancy. Available from: https://www.cometinitiative.org/Studies/Details/1670. Cited 2021 Jun 10.

47. COMET Initiative. COMET Database. Developing a core outcome set for planned mode of birth: a stakeholder consensus process. Available from: https://www.comet-initiative.org/Studies/Details/1648. Cited 2021 Jun 10.

48. COMET Initiative. COMET Database. Swedish perinatal core outcome set (SPeCOS). Available from: https://www.comet-initiative.org/Studies/Detai Is/1593. Cited 2021 Jun 10.

49. COMET Initiative. COMET Database. Developing a core outcome set for perinatal women with Generalized Anxiety Disorder (GAD): standardizing reported outcomes in clinical research. Available from: https://www. comet-initiative.org/Studies/Details/1823. Cited 2021 Jun 10.

50. Altoukhi S, Whitehead CL, Ryan G, Deprest J, Joyeux L, Gallagher K, et al. Development of a Core outcome set for fetal Myelomeningocele (COSMiC): study protocol. Trials. 2020;21(1):732.

51. COMET Database. The CO-OPT study: Core Outcomes in Obstetric Anal Sphincter Injury Prevention and Treatment. Available from: http://www. comet-initiative.org/studies/details/959. Cited 2020 Jan 31.

52. COMET Initiative. COMET Database. Network for preconception and early pregnancy randomised controlled trials. Available from: http://www. comet-initiative.org/studies/details/784. Cited 2020 Jan 31.

53. COMET Initiative. COMET Database. Constructing a core outcome set for iron deficiency and iron deficiency anaemia in pregnancy and postpartum. Available from: http://www.comet-initiative.org/studies/details/836. Cited 2020 Jan 31.

54. COMET Initiative. COMET Database. Constructing a core outcome set for immune thrombocytopenia in pregnancy. Available from: http://www. comet-initiative.org/studies/details/840. Cited 2020 Jan 31.

55. COMET Initiative. COMET Database. Developing a core outcome set for research in multiple pregnancies. Available from: http://www.comet-initi ative.org/studies/details/844. Cited 2020 Jan 31.

56. COMET Initiative. COMET Database. Core outcome set for pregnancy outcomes in pregnancies complicated by cancer (COSPOP). Available from: http://www.comet-initiative.org/studies/details/1098. Cited 2020 Jan 31.

57. COMET Initiative. COMET Database. Core outcome set for sickle cell disease in pregnancy. Available from: http://www.comet-initiative.org/ studies/details/1190. Cited 2020 Jan 31.

58. COMET Initiative. COMET Database. Core outcome set for rectovaginal fistula (RVF). Available from: http://www.comet-initiative.org/studies/ details/1082. Cited 2020 Jan 31.

59. COMET Initiative. COMET Database. Development of a core outcome set and identification of outcome measurement tools for interventions for prevention of stillbirth and care after stillbirth (International Collaboration for Harmonising Outcomes fOr Stillbirth resEarch: ICHOOSE). Available from: http://www.comet-initiative.org/studies/details/775. Cited 2020 Jan 31.

60. COMET Initiative. COMET Database. Developing a core outcome set (COS) for clinical trials on interventions for the detection and management of reduced fetal movements in pregnancy. Available from: http:// www.comet-initiative.org/studies/details/928. Cited 2020 Jan 31.

61. COMET Initiative. COMET Database. Development of a core outcome set for operative vaginal birth. Available from: http://www.comet-initiative. org/studies/details/1069. Cited 2020 Feb 3.

62. COMET Initiative. COMET Database. The development of a core outcome set for breastfeeding research. Available from: http://www.comet-initi ative.org/studies/details/1028. Cited 2020 Feb 3.
63. COMET Initiative. COMET Database. The composite adverse obstetric outcomes study (CAOOS). Available from: http://www.comet-initiative. org/studies/details/1154. Cited 2020 Feb 3.

64. COMET Initiative. COMET Database. A protocol for developing and implementing a core outcome set in ectopic pregnancy. Available from: http:// www.comet-initiative.org/Studies/Details/1492. Cited 2020 Feb 3.

65. D'Souza R, Hall C, Sermer M, Siu S, Silversides C. Development of a core outcome set for studies on cardiac disease in pregnancy (COSCarP): a study protocol. Trials. 2020;21(1):300.

66. D'Souza R, Villani L, Hall C, Seyoum M, Kingdom J, Krznaric M, et al. Core outcome set for studies on pregnant women with vasa previa (COVasP): a study protocol. BMJ Open. 2020;10(7):e034018.

67. Dadouch R, Faheim M, Juando-Prats C, Parsons J, D'Souza R. Development of a core outcome set for studies on obesity in pregnant patients (COSSOPP): a study protocol. Trials. 2018;19(1):655.

68. Doumouchtsis SK, Rada MP, Pergialiotis V, Falconi G, Haddad JM, Betschart C. A protocol for developing, disseminating, and implementing a core outcome set (COS) for childbirth pelvic floor trauma research. BMC Pregnancy Childbirth. 2020;20(1):376.

69. Initiative C. COMET Database. Developing a core outcome set for studies on women with invasive placentation. Available from: http://www. comet-initiative.org/studies/details/1127. Cited 2020 Jan 31.

70. Kgosidialwa O, Bogdanet D, Egan A, O'Shea PM, Biesty L, Devane D, et al. Developing a core outcome set for the treatment of pregnant women with pregestational diabetes-a study protocol. Trials. 2020;21(1):1017.

71. Killeen SL, O'Brien EC, Jacob CM, O'Reilly SL, Hanson M, McAuliffe FM. PREgnancy Nutrition: a protocol for the development of a Core Outcome Set (PRENCOS). Int J Gynaecol Obstet. 2019;147(2):134-9.

72. King A, D'Souza R, Teshler L, Shehata N, Malinowski AK. Development of a core outcome set for studies on prevention and management of pregnancy-associated venous thromboembolism (COSPVenTE): a study protocol. BMJ Open. 2020;10(7):e034017.

73. Prins JR, Holvast F, van't Hooft J, Bos AF, Ganzevoort JW, Scherjon SA, et al. Development of a core outcome set for immunomodulation in pregnancy (COSIMPREG): a protocol for a systematic review and Delphi study. BMJ Open. 2018;8(8):e021619.

74. Sankaran S, Ching-Soh M, Nelson-Piercy C. The urgent need for a multidisciplinary core outcome set for the reporting of obstetric antiphospholipid antibody syndrome. Trials. 2015;16(3):1.

75. Smith P, Cooper N, Dhillon-Smith R, O'Toole E, Clark TJ, Coomarasamy A. Core outcome sets in miscarriage trials (COSMisT) study: a study protocol. BMJ Open. 2017:7(11):e018535.

76. Smith V, Daly D, Lundgren I, Eri T, Begley C, Gross MM, et al. Protocol for the development of a salutogenic intrapartum core outcome set (SIPCOS). BMC Med Res Methodol. 2017;17(1):61.

77. Vergote S, De Bie F, Bosteels J, Hedrick H, Duffy J, Power B, et al. Study protocol: a core outcome set for perinatal interventions for congenital diaphragmatic hernia. Trials. 2021;22(1):1-7.

78. Viau-Lapointe J, D'Souza R, Rose L, Lapinsky SE. Development of a core outcome set for research on critically ill obstetric patients: a study protocol. Obstet Med (1753-495X). 2018;11(3):132-6.

79. Viau-Lapointe J, Lapinsky S, D'Souza R, Kfouri J, Rose L. Development of a core outcome set for studies of pregnant women requiring mechanical ventilation. J Evid Based Med. 2017;10:24.

80. Whitehouse KC, Kim CR, Ganatra B, Duffy JMN, Blum J, Brahmi D, et al. Standardizing abortion research outcomes (STAR): a protocol for developing, disseminating and implementing a core outcome set for medical and surgical abortion. Contraception. 2017;95(5):437-41.

81. Damhuis SE, Bloomfield FH, Khalil A, Daly M, Ganzevoort W, Gordijn SJ. A core outcome set and minimum reporting set for intervention studies in growth restriction in the NEwbOrN: the COSNEON study. Pediatr Res. 2021;89(6):1380-5.

82. Kelly LE, Shan F, MacVicar S, Czaplinksi E, Moulsdale W, Simpson S, et al. A core outcome set for neonatal opioid withdrawal syndrome. Pediatrics. 2020;146(1):e20200018.

83. Boers M, Brooks $P$, Simon LS, Strand V, Tugwell P. OMERACT: an international initiative to improve outcome measurement in rheumatology. Clin Exp Rheumatol. 2005;23(5 Suppl 39):S10-3. 
84. James Lind Alliance (JLA). Southampton: National Institute for Health Research, Evaluation, Trials and Studies Coordination Centre (NETSCC), James Lind Alliance. Available from: http://www.jla.nihr.ac.uk/. Cited 2020 Jun 10.

85. Kirkham JJ, Davis K, Altman DG, Blazeby JM, Clarke M, Tunis S, et al. Core Outcome Set-STAndards for Development: the COS-STAD recommendations. PLoS Med. 2017;14(11):e1002447.

\section{Publisher's Note}

Springer Nature remains neutral with regard to jurisdictional claims in published maps and institutional affiliations.

- fast, convenient online submission

- thorough peer review by experienced researchers in your field

- rapid publication on acceptance

- support for research data, including large and complex data types

- gold Open Access which fosters wider collaboration and increased citations

- maximum visibility for your research: over $100 \mathrm{M}$ website views per year

At BMC, research is always in progress.

Learn more biomedcentral.com/submissions 